\title{
Testing Einstein in Space: The Gravity Probe B Relativity Mission
}

\author{
John Mester and the GP-B Collaboration \\ Hansen Laboratory \\ Stanford University \\ Stanford CA, USA
}

\begin{abstract}
The Gravity Probe B Relativity Mission was successfully launched on April 20, 2004 from Vandenberg Air Force Base in California, a culmination of 40 years of collaborative development at Stanford University and NASA. The goal of the GP$\mathrm{B}$ experiment is to perform precision tests of two independent predictions of general relativity, the geodetic effect and frame dragging. On-orbit cryogenic operations lasted 17.3 months, exceeding requirements. Analysis of the science data is now in progress with a planned announcement of results scheduled for April 2007.
\end{abstract}

\section{Introduction}

Our present theory of gravity, Einstein's general relativity, is elegant, internally consistent and (so far) in agreement with observation. Yet, despite recent advances, the range of predictions tested and the precision to which experiments have tested the theory remain limited [1]. In addition, general relativity resists quantization, thwarting efforts to include the theory in a unified picture of the forces of nature. Nearly all attempts at unifying gravitation with the Standard Model result in a theory which differs form general relativity, and in particular, include additional vector or scalar couplings which potentially violate the Equivalence Principle $[2,3]$.

Space offers the opportunity for new tests of general relativity with improved precision [4]. The use of drag compensation, first demonstrated in flight by the Discos instrument on the Triad mission [5], to reduce air drag, magnetic torque, and radiation pressure disturbances enables a uniquely quiet environment for experimentation, one not limited by seismic noise. In the following we describe a space based fundamental physics experiment, the Gravity Probe B Relativity Mission.

\section{Gravity Probe B}

The GP-B spacecraft was launched from Vandenberg Air Force Base on April 20, 2004 on a Boeing Delta II two-stage rocket. Although the operation plan included a 30 day orbit trim procedure, the initial orbit injection was near perfect and placed the vehicle within 100 meters of its target near the Earth's poles.

The Gravity Probe B Relativity Mission is a space based experiment developed at Stanford University with oversight by the Marshall Space Flight Center and funding from the NASA Office of Space Science. GP-B will test two predictions of general relativity, the geodetic and frame dragging effects, by measuring the precession of gyroscopes in a $642 \mathrm{~km}$ high orbit around the earth. The philosophy of the experiment is to reduce disturbances to levels at which general relativistic effects should be manifest without large subtraction of Newtonian drifts.

The relativistic precession of a gyroscope in a circular orbit around the earth is given by:

$$
\bar{\Omega}=\left(\gamma+\frac{1}{2}\right) \frac{G M}{c^{2} R^{3}}(\bar{R} \times \bar{v})+\left(\gamma+1+\frac{\alpha_{1}}{4}\right) \frac{G I}{2 c^{2} R^{3}}\left[\frac{3 \bar{R}}{R^{2}} \cdot\left(\bar{\omega}_{e} \cdot \bar{R}\right)-\bar{\omega}_{e}\right]
$$




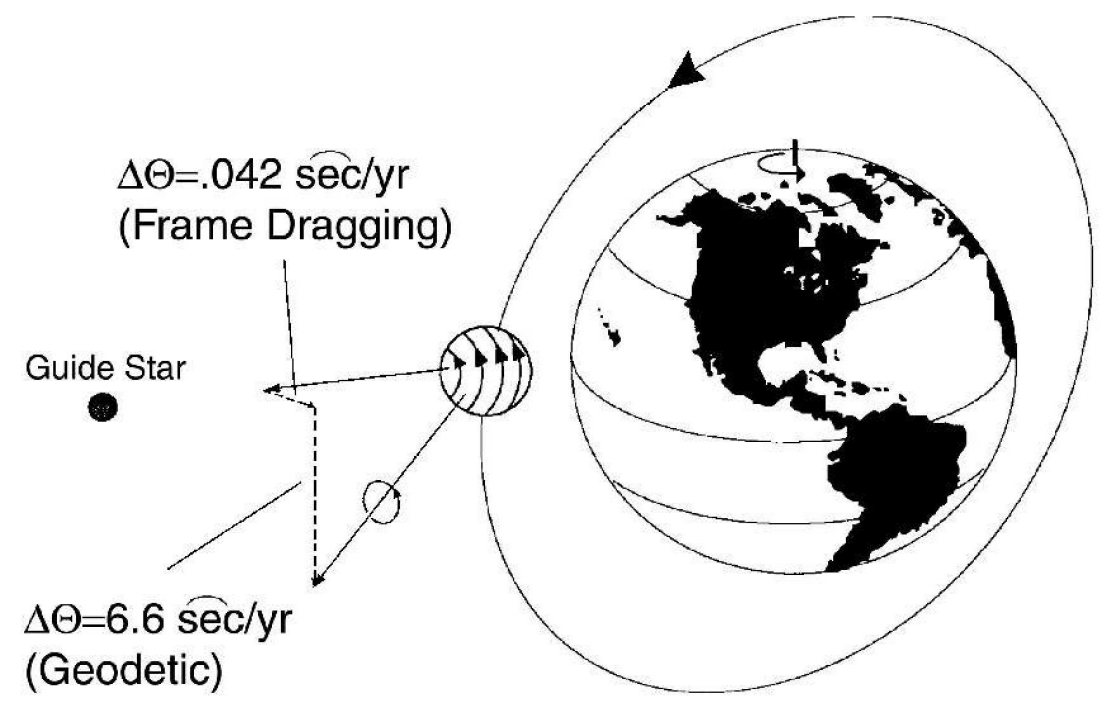

Figure 1: GP-B Concept

where $R$ is the position and $\mathrm{v}$ the orbital velocity of the gyroscope, $I, M$, and $\omega$ are the moment of inertia, mass and angular velocity of the earth, and $G$ is the gravitational constant. For generality we include the PPN parameters $\gamma$ and $\alpha_{1}$; in general relativity $\gamma=1$ and $\alpha_{1}=0$. The first term describes the geodetic precession, which arises from the curvature of spacetime due to the mass of the earth. General Relativity predicts that the spin direction of the gyroscope will change at the rate of 6.6 arcsec per year for a $642 \mathrm{~km}$ high, polar orbit. The second term, frame dragging or Lense-Thirring effect, represents the precession due to the dragging of the inertial frame by the rotation of the earth. General Relativity predicts the rate of procession of a Gravity Probe B gyroscope to be $0.042 \mathrm{arc} \mathrm{sec}$ per year $(42 \mathrm{marcsec} / \mathrm{yr})$. A polar orbit is chosen so the two precessions are orthogonal and can therefore be distinguished. Figure 1 depicts the direction of the two precessions.

\section{Experimental System Overview}

The small size of the relativity precessions requires that the experiment system have extreme measurement precision and that all sources of error be controlled. In order to achieve this requirement the experiment exploits the advantages of a near zero-g orbit in space and a near zero temperature in the experimental probe[6]. The experimental module consists of a helium dewar, which holds 2500 liters of superfluid helium, surrounding the experimental probe containing four gyroscopes, quartz block, and a star tracking telescope. The dewar is designed have an on orbit helium lifetime of greater than 16.5 months. Actual on-orbit lifetime was 17.3 months. The helium is maintained at a temperature of $1.8 \mathrm{~K}$ by means of a porous plug venting system and the boil off gas is used to power proportional thrusters used in drag free control. The thrusters keep the space craft centered around a gyroscope in free fall to produce residual accelerations at this gyroscope of less than $10^{9} g\left(g=9.8 \mathrm{~m} / \mathrm{s}^{2}\right)$. Three other gyroscopes are mounted within a rigid quartz block assembly. The quartz block provides precise positioning of the gyroscopes and the telescope, with cryogenic temperatures increasing mechanical stability. A series of windows provides an open line of sight out of the dewar. The star tracking telescope is used to point the spacecraft towards a guide star, providing a distant inertial reference with which to compare the gyro spin direction. 


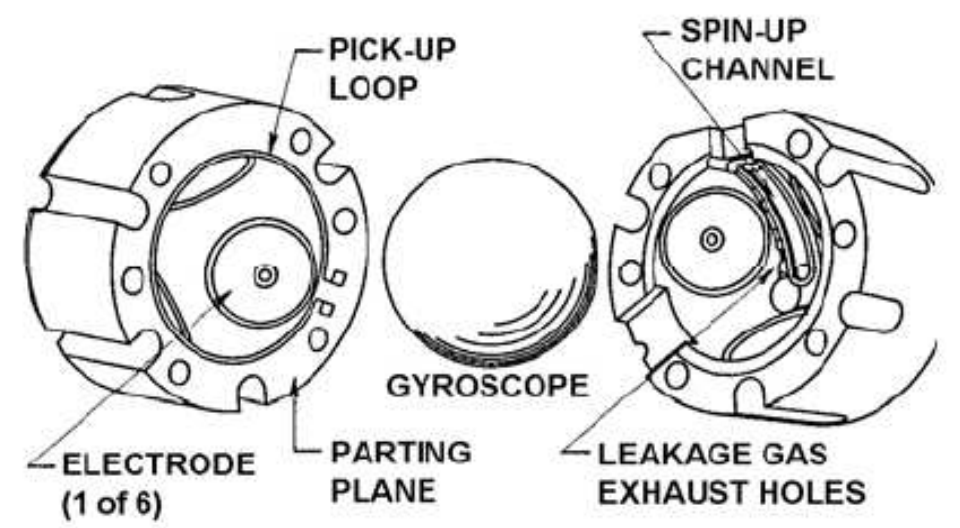

Figure 2: GP-B Gyroscope

The spacecraft rolls about the line of sight to the guide star with a period of 77.5 seconds. This averages off axis accelerations (which would contribute to Newtonian torques at the gyroscopes) to below $5 \times 10^{12} \mathrm{~g}$ and allows the gyroscope spin direction to be measured at roll frequency, eliminating DC measurement complications.

\section{Gyroscopes and Gyroscope Readout}

At the heart of the gravity probe $\mathrm{B}$ mission are the gyroscopes. Figure 2 gives a schematic, exploded view of a gyroscope. The gyroscope is comprised of a rotor, 3.8 $\mathrm{cm}$ in diameter, which spins freely within the spherical cavity of a quartz housing. Newtonian torques on the gyroscope are minimized by the drag free satellite system and by controlling rotor sphericity and homogeneity. Rotors are fabricated from fused quartz with density inhomogeneity of less than 2 parts per million and are ground and lapped to achieve peak-to-valley asphericity of less than $25 \mathrm{~nm}$. The rotors are coated with a 1.25 micron thick uniform layer of niobium which has a superconducting transition temperature of $9.2 \mathrm{~K}$. The niobium coating enables the rotor to be electrostatically suspended within the housing and provides a means for sensing the gyroscope spin direction, discussed below. The housing for the rotor, shown in two halves split by a parting plane, has 3 orthogonal pairs of electrodes used to suspend and sense the position the rotor. Once suspended, the rotors are spun-up by directing helium gas through the spin up channel. The helium gas is then pumped away to high vacuum to eliminate residual gas damping of the rotor.

The housing electrodes also provide a means of sensing rotor charge. Since the rotors are freely floating, charge can accumulate due mainly to cosmic ray, solar and South Atlantic Anomaly protons. A charge control system incorporates a UV fiber optic actuator that produces and controls photo electrons.

The gyroscope readout system must be capable of resolving changes in the rotor spin direction of less than 1 marcsec without producing interaction torques that could disturb that spin direction $[7,8]$. The low experimental operating temperature allows the properties of superconductivity to be exploited, both as the physical basis of the readout signal and in its detection. The readout signal is based on the magnetic field produced by the London moment of a rotating superconductor. When the superconducting niobium coated rotor is spun up, it develops a London magnetic moment aligned with its instantaneous spin axis. The London moment produces an equivalent magnetic field just outside the rotor of magnitude:

$$
B_{L}=1.14 \times 10^{7} \omega_{s} \text { Gauss }
$$


where $\omega_{s}$ is the spin angular velocity. The London field is measured using a dc SQUID (superconducting quantum interference device) magnetometer. On the parting plane between the two housing halves, there is a four turn superconducting loop that couples the London moment flux to the SQUID. The gyro spin axis is aligned close to the spacecraft roll axis, which lies in the parting plane, so the London moment produces a signal modulated at roll frequency. At a spin speed at 80 hertz, the London field equals $5.7 \times 10^{5}$ Gauss. Therefore, to resolve 1 marcsec changes in spin direction a field sensitivity of $2.8 \times 10^{13}$ Gauss is required. The noise performance of Gravity Probe B SQUID readout system met this requirement with margin, as discussed in the next section.

Such low field levels also dictate the need for extensive magnetic shielding. Ultra low dc fields of less than $10^{7}$ Gauss, required to minimize flux trapping in the rotor, are produced using the expanded superconducting lead shield technique [9]. This shield, coupled with extra internal superconducting shielding and an external cryoperm shield yield ac (roll frequency) field attenuation at the gyroscopes of greater than $2 \times 10^{12}$.

\section{Telescope and Guide Star Selection}

It is necessary to measure the spin direction of the gyroscopes relative to a distant reference frame, one not affected by the mass or spin of the earth. Therefore, a telescope is incorporated into the experimental module to track the position of a guide star. The star tracking telescope is of the folded Schmidt-Casagranian type with a $14.4 \mathrm{~cm}$ diameter aperture. It is constructed out of fused quartz and has an overall physical length of $50 \mathrm{~cm}$. Two focused images are formed on the edge of roof prisms by splitting the incoming starlight with a beamsplitter. The edges of the roof prisms are perpendicular, providing two axis readout. Each prism divides the star image into two partial images whose intensities are determined using cryogenic silicon photo detectors and cryogenic preamplifiers. The relative intensities of the prism-split images determine the direction of the line of sight to the guide star. Using this signal, the spacecraft attitude is controlled to point in the direction of the guide star.

An important factor in reaching design measurement accuracy is the selection of the guide star to act as an adequate inertial reference. Uncertainties in the proper motion of the guide star propagate directly as experimental error and therefore the guide star proper motion needs to be known to high precision. Review of candidate stars led to the selection of HR8703, which is an optical star of 5.69 magnitude and is also radio star. Observations by the Harvard Smithsonian Astronomical Observatory using VLBI have establish sub 0.1 marssec/yr proper motion uncertainties [10]. VLBI observations are continuing to further reduce these uncertainties.

In parallel with space vehicle development activities, a mission operations center (MOC) was commissioned at Stanford University. The MOC conducted command and control throughout mission lifetime. The Wallops Island IONet provides communication with the TDRRS satellite communications network and ground stations at Svalbard, Norway and Poker Flat, Alaska.

\section{On-Orbit Operations and Performance}

On-orbit operations for GP-B lasted 17.3 months, from launch until the liquid helium cryogen was depleted. The first phase of the mission, Initial Operation and Checkout (IOC) consisted of 4.3 months of experiment setup. This was followed by 11.6 months of science data collection, and 1.4 months of post-science calibrations.

During the IOC phase many operations were required to configure the experiment for science. These included setting up the attitude and translation control system and spinning up the gyros. Initial coarse attitude was achieved using magnetic sensors and star trackers to set the spacecraft roll to a convenient operating period and 
to point the spacecraft to within the 60 arc-sec field of view of the telescope. Once this was realized the signal form the telescope could be included in the sensors array. Because direction to the guide star lies in the plane of the spacecraft orbit, the star is eclipsed each time the vehicle travels behind the earth. Therefore the star has to be re-acquired each orbit. After tuning of control parameters, typical drive-in times of less than 60 seconds were achieved. RMS pointing noise was less than 200 marc-sec, pointing noise at roll frequency was $\sim 5$ marc sec.

Before gyro spin operations could proceed the gyro suspension system (GSS) electronics were activated to levitate the gyro rotors within their housings. Typical on-orbit RMS gyro position measurement noise was $0.45 \mathrm{~nm}$. The control effort to keep the gyros centered provides a measure of the gyro acceleration environment. These signals were used as input to the translation control system enabling dragfree operation. Despite the loss of 2 of the 16 helium proportional thrusters, drag free performance met requirements, achieving cross track acceleration levels of $<4 \times 10^{12} \mathrm{~g}$ over the frequency range of $0.01 \mathrm{mHz}$ to $0.1 \mathrm{~Hz}$.

In order to reduce measurement noise and disturbance torques, gyro charge and trapped magnetic flux had to be minimized. The trapped flux reduction procedure consisted of heating the gyro rotors above the $\mathrm{Nb}$ superconducting transition temperature and then carefully cooling down within the ultra-low field environment provided by the superconducting shield system. All four gyros met requirements with trapped flux levels between 0.2 to 3 micro-Gauss. Rotor charge was controlled throughout the mission using a system that included the GSS sensing and UV fiber optic actuation mentioned above. After initial levitation (from which the gyros acquired charges in the range of 100 s of milli-Volts), typical charging rates throughout the mission were $0.1 \mathrm{mV}$ per day. Several discharges were performed during the mission.

Gyro spin-up was accomplished by commanding a helium gas reservoir system to flow helium through the spin up channels of each gyro. In order to keep background pressures low, (high pressure could interfere with gyro suspension) the gyros were spun up one at a time and helium was exhausted to space through $15 \mathrm{~cm}$ diameter valves. Spun gyros would slow down during the spin-up of subsequent gyros. Final gyro spin rates were in the range $\simeq 60$ to $80 \mathrm{~Hz}$, see table 1 . After the final spinup the probe residual pressure was reduced to below $10^{11}$ Torr using a cryogenic bake-out procedure. This resulted in characteristic spindown period that exceeded requirements. The final procedure of the IOC phase was the adjustment of the gyro

\begin{tabular}{|c|c|c|}
\hline & $\begin{array}{c}\text { Spin rate } \\
(\mathrm{Hz})\end{array}$ & $\begin{array}{c}\text { Spindown period } \\
\text { (years) }\end{array}$ \\
\hline Gyro 1 & 79.4 & 15,800 \\
\hline Gyro 2 & 61.8 & 13,400 \\
\hline Gyro 3 & 82.1 & 7,000 \\
\hline Gyro 4 & 64.9 & 25,000 \\
\hline
\end{tabular}

Table 1: Gyro spin rates and 1/e spindown periods

spin axes to point within 10 arc-sec of the guide star line of site. This is required to reduce cross track torques and readout system errors. Spin axis alignment was accomplished by applying large voltages to the gyro housing electrodes in a carefully controlled sequence. This procedure also yielded an initial calibration of suspension system induced torques.

With the completion of experiment set-up, the mission entered science data phase in August, 2004. One of the main activities during the science phase was the measurement of the London moment signals from each gyro. A typical London Moment data curve is shown in figure 3 . The high frequency oscillation is due to spacecraft roll, a $77.5 \mathrm{sec}$ period. The broad envelope is due to guide star aberration. 


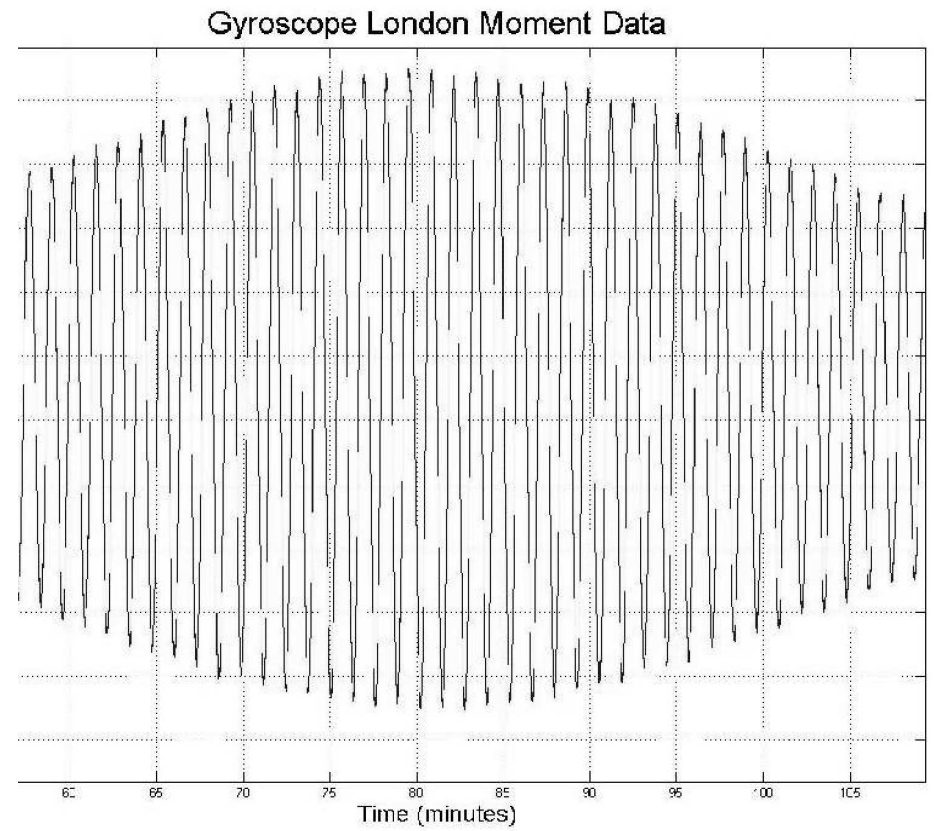

Figure 3: Gyro London Moment readout signal

The transverse velocity of the spacecraft causes a shift in the perceived guide star direction relative to the true, nearly inertially fixed location. The aberration has two components, one at the spacecraft orbit frequency and one at the annual frequency of the earth revolving around the sun. These well known amplitudes, (5.1856 arcsec orbital, 20.4958 arc-sec annual) provide an important tool to calibrate the gyro readout/telescope readout measurement scale factor.

After the 350 day, 6000 orbit science data phase the mission entered a post calibration stage. During this period operations were conducted to purposely increase disturbances as a means of characterizing systematic error. This included pointing the spacecraft toward other guide stars to increase cross axis gyro torques as well as varying GSS control parameters. This phase lasted 56 days until September 29, 2005 when the last of the helium cryogen boiled off.

\section{Data Analysis}

With the completion of cryogenic operations the mission has entered the data analysis phase. The spacecraft communication and operations system recovered $>99 \%$ of all the data taken during the mission. These 1.5 Terabytes of data include the so called science data the SQUID and Telescope readout signals - as well as engineering data form over 700 onboard sensors. In addition to the nominal data rate of 0.1 to $1 \mathrm{~Hz}$, 200-2200 Hz snapshot data from key systems were also recorded and down-linked.

It is anticipated that analysis will continue into early 2007. After the analysis is completed, the separately measured proper motion of the guide star will be subtracted from the gyroscope precession rate estimates to give the final experiment result. The final results and release of data are scheduled for April 2007. 


\section{References}

[1] C.M. Will, The confrontation between general relativity and experiment, Astrophysics and Space Science 283, no.4 (2003), 543-52.

[2] T. Damour, A.M. Polyakov, The string dilaton and a least coupling principle, Nuclear Physics B 25, vol.B423, no.2-3 (July 1994), 532-58.

[3] I. Antoniadis, S. Dimopoulos, A. Giveon, Little string theory at a TeV, JHEPJournal of High Energy Physics (2001).

[4] G.M. Keiser, General Relativity Experiments in Space, Adv. In Space Research 32, no.7 (2003), 1397-1400.

[5] D.B. DeBra, Disturbance compensation system design, APL Technical Digest 12, no.2 (April-June 1973), p.14-26.

[6] S. Buchman et. al., The Gravity Probe B relativity mission, Advances in Space Research 25, no.6 (2000), 1177-80.

[7] J.C. Mester, J.M. Lockhart, B. Muhlfelder, D.O. Murray, M.A. Taber, Ultralow magnetic fields and Gravity Probe B gyroscope readout, Advances in Space Research 25, no.6 (2000), 1185-8.

[8] J.M. Lockhart, B. Muhlfelder, G.M. Gutt, M. Luo, R.C. Clappier, T.R. McGinnis, G.R. Smith, Optimization of a SQUID system for space, IEEE Transactions on Applied Superconductivity 7, no.2, pt.3, (June 1997), 2534-7.

[9] M. Taber, D. Murray, J. Lockhart, D. Frank, D. Donegan, Production of Ultralow Magnetic Fields for Gravity Probe B, Advances in Cryo. Eng. 39A (1993), 161170.

[10] S. Wang, D.-H. Gwo, K.A. Bower, L.W. Huff, J.A. Lipa, Status of the cryogenic telescope and guide star for Gravity Probe B, Advances in Space Research 25, no.6 (2000), 1189-92. 